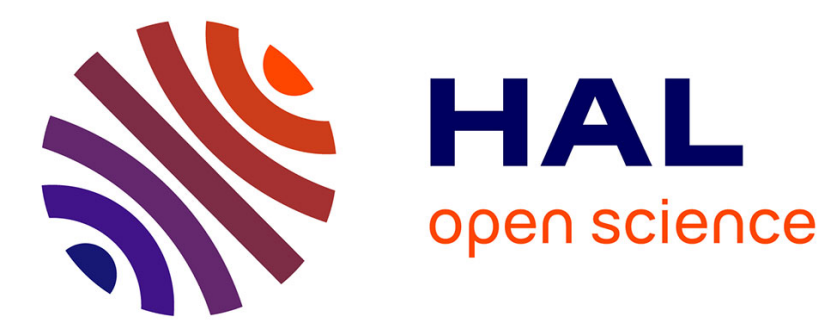

\title{
Wikipédia au prisme de l'épistémologie sociale et des études des sciences
}

Pierre Willaime, Alexandre Hocquet

\section{To cite this version:}

Pierre Willaime, Alexandre Hocquet. Wikipédia au prisme de l'épistémologie sociale et des études des sciences. Cahiers philosophiques, 2015, 2 (141), pp.68-86. halshs-01128512

\section{HAL Id: halshs-01128512 \\ https://shs.hal.science/halshs-01128512}

Submitted on 11 Mar 2015

HAL is a multi-disciplinary open access archive for the deposit and dissemination of scientific research documents, whether they are published or not. The documents may come from teaching and research institutions in France or abroad, or from public or private research centers.
L'archive ouverte pluridisciplinaire HAL, est destinée au dépôt et à la diffusion de documents scientifiques de niveau recherche, publiés ou non, émanant des établissements d'enseignement et de recherche français ou étrangers, des laboratoires publics ou privés.

\section{(c)(1)}

Distributed under a Creative Commons Attribution| 4.0 International License 


\title{
Wikipédia au prisme de l'épistémologie sociale et des études des sciences.
}

\author{
Pierre Willaime et Alexandre Hocquet \\ Laboratoire d'Histoire des Sciences et de Philosophie \\ Archives Henri Poincaré \\ UMR 7117 CNRS / Université de Lorraine
}

Février 2015

\section{Table des matières}

1 Épistémologie sociale et numérique 2

1.1 Informatique et culture numérique . . . . . . . . . 3

1.2 Logiciels libres et édition participative de contenus . . . . . . 4

1.3 L'épistémologie sociale comme changement de paradigme épistémique ................... 7

2 Wikipédia, mouvement du logiciel libre et matérialité $\quad 11$

2.1 Études des sciences et techniques . . . . . . . . . . . . 12

2.2 Influences mutuelles . . . . . . . . . . . . . . . . . . 13

2.3 Sphère publique . . . . . . . . . . . . . . . . . . . . . 14

2.4 Public récursif . . . . . . . . . . . . . . . 15

2.5 Wikipédia en tant que public récursif . . . . . . . . 16

2.6 Liberté brumeuse . . . . . . . . . . . . . . . . . 17

$\begin{array}{lll}3 & \text { Conclusion } & 19\end{array}$ 
Une grande partie des changements produits par l'informatique et le numérique concerne notre manière d'acquérir des connaissances. Wikipédia est ainsi devenue une source très populaire d'information. Dans cet article, nous nous proposons d'analyser en quoi les possibilités techniques introduites par l'informatique personnelle et le Web influencent certaines questions de philosophie de la connaissance. La première partie étudie l'influence de la technique et de modèles participatifs introduits par le mouvement des logiciels libres sur l'épistémologie. L'informatique contribue à motiver une épistémologie dite 《sociale» attentive aux systèmes d'interactions et au caractère collectif de la connaissance. La deuxième partie montre, à l'aide des STS (Science and Technology Studies), que les outils technologiques à notre disposition ne sont pas neutres mais sont, au contraire, chargés politiquement. Ainsi, la structure et le modèle épistémique de l'outil influencent politiquement son contenu et le type d'interaction et vice versa.

\section{1 Épistémologie sociale et numérique}

Dans cette première partie, nous nous intéressons aux changements épistémiques qu'accompagnent l'émergence d'une culture numérique. Il semble possible de détacher trois thèses principales sur cette question : soit la culture numérique modifie profondément ce que nous appelons « connaissance » et en nécessite une redéfinition (1) soit elle met simplement en lumière des aspects de la connaissance auparavant minorés et délaissés (2) soit, enfin, elle ne joue aucun rôle épistémique et n'est qu'un support de l'information (3). Dans la suite de cet article, nous défendrons principalement la seconde thèse. Cependant, nous soutiendrons que la mise en lumière de nouveaux aspects de la connaissance s'accompagne forcément de nouvelles pratiques et d'une redéfinition conceptuelle. Ainsi, il semble que la défense de la seconde thèse conduit également à soutenir, au moins partiellement, la première. Selon nous, l'informatique personnelle et la toile d'araignée mondiale (« World Wide Web ») permettent à la fois de prendre conscience d'une dimension collective et sociale de la connaissance (étudiée aujourd'hui par l'épistémologie sociale) et de remettre en cause certaines théories épistémiques.

De manière plus ou moins consciente, le recours à l'informatique est devenu quasiment automatique pour la recherche d'un certain type d'information et l'acquisition de certaines connaissances. Si, par exemple, vous vous demandez quelle est la capitale du Burkina Faso, vous allez très probablement utiliser Internet et trouver la réponse à votre question sur Wikipédia. L'utilisation d'un moteur de recherche vous conduira en effet à la page « Ouagadougou » de l'encyclopédie libre. Ces nouveaux processus de recherches et d'acquisition de connaissances ne sont pas anodins. Dans la lignée de Berry (2012), nous soutiendrons que le numérique devient une des conditions de possibilité de la connaissance. La médiation des acteurs non-humains 
tels les ordinateurs et les réseaux doit être prise en compte dans les formations de connaissance. Le numérique nous pousse à questionner la structure épistémique de ses outils et les conséquences de leur usage.

\subsection{Informatique et culture numérique}

Tout d'abord, précisons pourquoi nous parlons de «numérique » et pas d' " informatique ». Comme le souligne Doueihi (2013), les termes ne sont pas du tout synonymes. L'informatique fait habituellement référence à ses applications pratiques, c'est-à-dire à un ensemble d'outils computationnels. Ces outils, bien que n'étant pas neutres, ne s'accompagnent pas automatiquement de pratiques bien définies. Le numérique rassemble les manières, diverses dont nous utilisons les possibilités techniques informatiques à notre disposition. Les pratiques numériques populaires s'apparentent alors à ce que l'on peut appeler une « culture numérique ». Par conséquent, l'informatique n'est qu'une partie du numérique et la culture numérique peut s'émanciper des outils informatiques et se retrouver dans d'autres champs culturels.

Il est important de noter ici que l'informatique ne doit pas pour autant être limitée à ses applications pratiques. Nous aurions tort, en effet, de la concevoir comme une science appliquée dont le développement répondrait uniquement à des préoccupations techniques. Au contraire, une «philosophie de l'informatique » qui fournirait une analyse conceptuelle de ses objets et processus semble nécessaire. La création d'un langage de programmation ou d'une machine de Turing demande avant tout de définir un ensemble de concepts ontologiques et des activités de programmation. Comme Mélès le souligne (2014, p.182) l'informatique subit le préjugé propre aux sciences de l'ingénieur : « de ce qu'elles sont applicables, on croit pouvoir les conclure empiriques ». Nous pouvons donc retenir deux acceptions du terme « informatique » :

1. la première, plus courante, réfère aux possibilités techniques permises par l'outil informatique et est attachée à l'objet ordinateur,

2. la deuxième comprend « informatique » comme une activité philosophique de création d'ontologies et de définition de concepts tels que « fichier » ou « processus » par le biais de la programmation.

Nous choisissons donc ici de parler de numérique et ce terme fait référence à la manière dont nous utilisons l'outil informatique (première définition) ${ }^{1}$. Nous avons noté que le numérique, défini donc comme ensemble de pratiques d'appropriation d'outils computationnels, n'est pas réductible aux outils informatiques. Néanmoins, deux possibilités techniques jouent un rôle important dans le développement des cultures numériques.

1. Bien sûr, l'ontologie sous-jacente d'un outil informatique n'est pas sans lien avec les usages de cet outil. 
1. C'est tout d'abord l'accessibilité de l'information permise par sa numérisation qui introduit de nouveaux usages. Avec la dématérialisation, il est maintenant possible de dupliquer des contenus sans pour autant déposséder quelqu'un. Il ne s'agit plus alors de se dessaisir de tel ou tel livre pour en permettre la lecture à d'autres mais d'en copier le contenu en autant d'exemplaires que nécessaires pour que chacun puisse le lire en même temps. Les biens numériques sont anti-rivaux ${ }^{2}$ selon le néologisme introduit par Weber (2004). De plus, avec l'élaboration des protocoles $\mathrm{TCP} / \mathrm{I}^{3}$ et l'apparition d'Internet, les contenus numériques peuvent s'échanger facilement et rapidement.

2. L'informatique ne se contente pas de rendre la connaissance plus accessible, elle peut également jouer sur sa formation. En effet, avec le « Web 2.0 », les visiteurs d'un site web peuvent agir sur son contenu et ne plus être simple spectateurs. Ainsi, la gestion du contenu n'est plus réservée aux administrateurs qui ont accès aux serveurs mais peut être participative et ouverte à des non-techniciens.

On peut parler de « révolution informatique » comme le veut ce volume lorsque nous mesurons l'impact des possibilités techniques telles que la duplication des contenus sans dépossession, leur partage via Internet, la contribution participative avec le « Web $2.0 »$, etc. Cependant, nous aurions tort de considérer ces changements comme neutres. Leur portée réside dans la nouvelle conception du travail intellectuel et de la connaissance qu'ils véhiculent.

\subsection{Logiciels libres et édition participative de contenus}

Dès la fin des années 1960, les possibilités techniques permises par l'informatique questionnent le mode de fonctionnement des contenus numériques. Cela commence avec les logiciels. Dans les années 1960, une grande partie du développement était réalisée dans des laboratoires universitaires ou des entreprises par des scientifiques et des ingénieurs. Le logiciel faisait partie intégrante de l'ordinateur sur lequel il était conçu. La portabilité des logiciels entre deux machines était quelque chose de très rare, et l'idée de l'échange du logiciel, et encore moins de la vente ou de la licence, ne venait jamais à l'esprit de leurs concepteurs, qu'ils appartenaient au monde académique

2. Un bien anti-rival est, selon Weber, un bien dont l'usage n'est pas privatif par nature. Certains biens matériels sont généralement anti-rivaux : l'usage d'un lampadaire, par exemple, ne s'accompagne pas de l'indisponibilité de ce bien pour autrui (sauf si un nombre trop important de personnes veulent en profiter). Mais c'est avec la dématérialisation des biens que ce concept prend tout son sens : nous pouvons dupliquer un livre numérique un nombre indéterminé de fois sans pour autant en perdre l'usage.

3. TCP (Transmission Control Protocol) et IP (Internet Protocol) sont deux protocoles fondateurs d'Internet qui régissent le transfert des données. 
ou au monde industriel (Von Krogh 2003). Ce n'est qu'à partir de la fin des années 1960 que le logiciel est devenu un produit qui pouvait être acheté séparément du matériel.

C'est en 1967 que l'expression « software crisis » (la crise du logiciel) est apparue pour la première fois (Ensmenger 2010, Mahoney 2008). Les calendriers et les coûts de développement de logiciels ont explosé, les erreurs (les fameux bugs logiciels) étaient de plus en plus difficiles à localiser et à corriger à cause de la complexité croissante des codes, et les révisions des logiciels de plus en plus difficiles à mettre en œuvre. Comparée à l'évolution rapide du matériel, le développement des logiciels était de plus en plus embourbé (Aspray 2002). Comme le dit Ensmenger, au milieu des années 1980, la crise du logiciel n'était plus un tournant, c'était un mode de vie (Ensmenger 2010). Le point critique de l'industrie informatique était devenu le logiciel. Le développement du PC a ouvert la voie à une industrie du logiciel, puisqu'on disposait maintenant d'un matériel standardisé, diminuant ainsi le problème de la portabilité (c'est-à-dire la tâche de faire un travail de logiciel sur différents ordinateurs ou des systèmes d'exploitation différents). Dans les années 1980, la majorité de l'activité et des bénéfices de l'industrie informatique étaient dans le secteur des logiciels pour PC, ce qui a conduit à une concentration de l'industrie en quelques grands acteurs (Campbell-Kelly 2004). IBM, constructeur de matériel, était la société dominante dans le secteur de l'informatique des années 1960 et 1970 ; la société de logiciels Microsoft est devenue dominante dans les années 1990 (Aspray 2002). Cette concentration, cette situation de marché de type « winner takes all » a conduit à des stratégies industrielles pour rendre leurs clients captifs. Partager le logiciel n'était plus du tout une tactique industrielle pertinente (CampbellKelly 2004).

Les logiciels deviennent progressivement soumis à des licences restrictives qui empêchent leurs modifications et leur distribution. Une problématique émerge alors : pourquoi payer pour un logiciel qui peut être dupliqué à volonté? Plus encore, pourquoi interdire la modification des logiciels alors que l'utilisateur peut être compétent et à même de corriger un bug qui l'affecte?

Rapidement, un mouvement militant pour la « libération » des logiciels va se développer autour de figures telles que Richard Stallman (Cf. Stallman, Williams, and Masutti 2010) et la création de la Free Software Foundation. L'objectif de cette organisation est de promouvoir le logiciel libre qu'elle définie de la sorte :

La définition du logiciel libre selon la Free Software Foundation (https ://gnu.org/philosophy/free-sw.html) :

« Un programme est un logiciel libre si vous, en tant qu'utilisateur de ce programme, avez les quatre libertés essentielles : 
1. la liberté d'exécuter le programme comme vous voulez, pour n'importe quel usage (liberté 0) ;

2. la liberté d'étudier le fonctionnement du programme, et de le modifier pour qu'il effectue vos tâches informatiques comme vous le souhaitez (liberté 1 );

3. l'accès au code source est une condition nécessaire; la liberté de redistribuer des copies, donc d'aider votre voisin (liberté $2)$;

4. la liberté de distribuer aux autres des copies de vos versions modifiées (liberté 3); en faisant cela, vous donnez à toute la communauté une possibilité de profiter de vos changements; l'accès au code source est une condition nécessaire.

Un programme est un logiciel libre s'il donne toutes ces libertés aux utilisateurs de manière adéquate. Dans le cas contraire, il est non libre. Bien que nous puissions faire une distinction entre différents schémas de distribution non libres, en quantifiant ce qui leur manque pour être libres, nous les considérons tous comme équivalents dans leur manque d'éthique. »

Derrière ce mouvement des logiciels libres se trouve l'idée que nous ne devons pas continuer à fonctionner selon l'ancien paradigme prénumérique. $\mathrm{Au}$ contraire, nous devons profiter des possibilités techniques nouvelles et ne pas les limiter artificiellement.

Pourquoi permettre le libre accès et la modification des contenus? Parce que ceux-ci ne sont plus produits par un seul individu « expert » mais par une multitude travaillant de manière participative à l'aide du Web 2.0. La culture numérique est centrée sur la foule et non plus l'expert. Pour reprendre le sous-titre du célèbre ouvrage de Surowiecki (2004) : « the many are smarter than the few ». Les contenus ne sont plus alors produits selon une structure fortement hiérarchisée donnant tout pouvoir à une minorité d'《 experts ». Considérés comme des biens communs, ils font l'objet d'un travail collaboratif et soudent une communauté.

Restreignons maintenant notre propos aux connaissances. L'exemple le plus parlant d'une communauté épistémique reposant sur un logiciel libre est l'encyclopédie Wikipédia. Dans celle-ci tout un chacun peut modifier des articles et l'enrichir. La fiabilité des informations est assurée par le nombre de contributeurs qui, même s'ils ne sont peut-être pas des " experts », vont pouvoir corriger les erreurs et partager leurs connaissances. Ainsi, l'individu doit nécessairement se dessaisir des connaissances qu'il partage et accepter de les voir modifier. Le succès de Wikipédia nous indique que ce modèle épistémique participatif fonctionne; sans doute est-il plus à même de recenser des connaissances « communes » et est-il mis plus en difficulté dans certains 
champs techniques, mais Wikipédia n'en est pas moins considérée, aujourd'hui, comme une source de connaissance très populaire. Les alternatives à l'encyclopédie libre basées sur l'ancien système vertical plaçant l'expert au centre de la production de connaissances (Citizendium, Veropedia) n'ont pas réussi à reproduire le succès de Wikipédia. Le simple constat de l'usage (et la dépendance) de nos sociétés modernes à des sources d'informations participatives sur le Web telle que Wikipédia nous indique que notre conception de la connaissance a changé avec l'avènement de la micro-informatique et du Web 2.0.

Nous avons parlé de l'ancien paradigme prénumérique, précisons maintenant ce que nous entendons par ce terme. Ce paradigme se caractérise tout d'abord par son caractère asymétrique. Les interactions épistémiques y sont en effet réalisées dans un sens bien précis : d'une personne considérée comme experte vers le novice. Avant le développement d'Internet et des communautés épistémiques en ligne, il fallait, pour acquérir des connaissances s'en remettre à des autorités épistémiques plus ou moins légitimes. La relation n'est pas symétrique car l'expert n'attend rien des novices (qui ne peuvent d'ailleurs souvent pas le contacter). Au contraire, la culture numérique est fondamentalement symétrique et égalitaire. Pour prendre l'exemple de Wikipédia, chaque contributeur est considéré comme un expert potentiel. À ce titre, il peut intervenir (anonymement ou pas) et modifier l'encyclopédie. Wikipédia présuppose ainsi que les contributeurs sont de bonne foi et que les erreurs éventuelles seront corrigées par d'autres contributeurs (principe d'autocorrection). Nous retrouvons une conception de la connaissance par témoignage proche du principe de véracité de Thomas Reid (selon lequel nous sommes naturellement enclins à dire la vérité). Plus largement, les dispositifs techniques propres à la culture numérique semblent mettre en avant le caractère participatif et collectif de l'activité épistémique.

\subsection{L'épistémologie sociale comme changement de paradigme épistémique}

Dans la philosophie de la connaissance (analytique) contemporaine, un courant récent entend prendre en compte le caractère participatif de la connaissance : l'« épistémologie sociale ». Nous parlerons ici de l'épistémologie sociale goldmanienne telle que présentée dans Knowledge in a Social World (Goldman 1999). Cette étude de la dimension sociale de la connaissance analyse les interactions entre les individus. L'objectif est de déterminer quelles pratiques sont préférables pour l'acquisition de connaissances. Pour Goldman, plus une pratique sociale conduit à des croyances vraies, plus cette pratique peut être considérée comme «fiable». Son épistémologie sociale est donc basée sur le fiabilisme, ce système épistémique définissant la connaissance par la fiabilité du processus qui lui permet d'émerger (cf. Goldman 1979). 
L'épistémologie sociale est avant tout une réaction au caractère excessivement individualiste de la philosophie de la connaissance. On considère en effet (trop) souvent la connaissance du point de vue d'un individu isolé pratiquant l'introspection pour s'assurer de ses croyances. La définition classique de la connaissance issue du Théétète nous dit qu'une croyance est connaissance si elle est vraie et si elle est justifiée; de manière assez naturelle, ce critère de justification a souvent été compris subjectivement : j'ai une connaissance si je peux justifier ma croyance (vraie). La dérive d'une telle conception de la justification est de considérer le sujet comme la seule entité pertinente pour évaluer nos croyances. Dès lors, la place de l'interaction sociale dans la formation des croyances est minorée. Cet « internalisme » épistémique est problématique. Philosophiquement, il suppose un accès privilégié de l'individu à ses états mentaux et la capacité de croire à volonté (volontarisme doxastique) selon la possession de la justification. Pourtant, Wittgenstein a critiqué l'hypothèse d'un langage qui serait uniquement " privé » et nous pouvons constater la persistance de certaines de nos croyances apparemment injustifiées. De manière plus concrète, l'épistémologie sociale se détache de l'internalisme dans sa volonté d'étudier des pratiques sociales relatives à la connaissance. Son objectif est de se saisir de sujets auparavant délaissés par la philosophie de la connaissance et laissés à la sociologie. Goldman veut développer une étude normative des pratiques épistémiques qui se distinguerait à la fois d'une épistémologie historique individualiste et du descriptivisme de la sociologie de la connaissance.

Une précision importante semble ici nécessaire : l'épistémologie sociale suppose une dichotomie forte entre une épistémologie individuelle ou individualiste et une épistémologie dite « sociale ». D'aucuns pourraient alors reprocher à l'épistémologie sociale goldmanienne de présupposer que la formation de la connaissance n'était pas « sociale» auparavant. Entendons-nous bien : c'est uniquement le manque de prise de conscience de cette formation sociale des connaissances que l'épistémologie sociale déplore et entend mettre en avant. Si Descartes est souvent cité comme l'archétype d'un épistémologue individualiste, c'est bien entendu pour la méthode de fondation de la connaissance introspective et uniquement centré sur l'individu qu'il propose. Ce sont ses propositions philosophiques qui négligent le rôle épistémique de l'interaction et du groupe dans la formation des connaissances, pas sa pratique personnelle qui était elle formée de multiples échanges (épistolaires en particulier) et influences. Cela étant dit, comme nous l'avancions au début de cet article, une prise en compte de l'importance épistémique des interactions sociales s'accompagne de conséquences sur la nature de la connaissance et de nouvelles pratiques épistémiques.

Les nouvelles pratiques propres à l"informatique et à l'utilisation du Web sont des objets d'étude rêvés pour l'épistémologie sociale. Tout d'abord, ces nouveaux outils illustrent le caractère nécessairement «social » de la connaissance. Les communautés épistémiques sur la toile sont régies par 
des ensembles de règles diverses qui sont autant de manières différentes de collaborer. Avant Internet, on pouvait encore se faire une représentation idéalisée des processus épistémiques comme échappant aux biais sociaux, comme étant produit par un seul individu; avec le succès de communautés comme celle de Wikipédia, on a l'illustration concrète d'échanges de connaissances qui fonctionnent car libres et participatifs. Il n'est donc pas étonnant que les philosophes de la connaissance commencent à s'intéresser à l'étude des médias numériques. De plus, l'aspect normatif de l'épistémologie sociale permet d'étudier les technologies de l'information et de la communication (TIC) comme rassemblant des processus d'acquisition de connaissances. Les TIC remplissant alors une fonction normative d' " assistance à la vérité » (Conein 2007, p.192).

Il nous faut préciser ici un point important : ce n'est pas parce que l'épistémologie sociale est sensible aux développements des cultures numériques (car illustrant le caractère « social » de la connaissance) qu'elle les considère forcément comme positives. Goldman lui-même dresse un portrait critique de ce qu'il appelle « la blogosphère » (Goldman 2008). L'épistémologie sociale ouvre de nouveaux champs d'étude mais n'impose pas de thèses prédéfinies. Ainsi, David Coady $(2011,2012)$ défend cette « blogosphere » face aux médias conventionnels en lui reconnaissant un certain nombre d'avantages épistémiques. Coady va même jusqu'à renforcer l'ancrage de l'épistémologie dans l'analyse de pratiques en défendant une "épistémologie appliquée » (Coady 2012). En effet, ce n'est pas parce qu'une épistémologie est sociale et donc anti-individualiste qu'elle est appliquée; elle peut rester relativement théorique et ne pas s'intéresser outre mesure à des études précises de cas.

Les nouvelles possibilités offertes par l'informatique ne sont pas qu'une illustration de la construction nécessairement communautaire des connaissances, elles sont surtout un moyen de remettre en cause certaines positions épistémiques. Si l'on prend l'exemple de Wikipédia, ou plutôt de l'épistémologie sociale de Wikipédia baptisée «Wikipistemology ${ }^{4}$. par Don Fallis (2008), le modèle totalement ouvert de l'encyclopédie a souvent été critiqué. L'absence de reconnaissance d'une expertise qui conditionnerait la rédaction d'un article a conduit certains auteurs à questionner la fiabilité de cette encyclopédie libre. Cette communauté sert donc d'exemple empirique pour dresser une comparaison entre un système épistémique ouvert, qui prend le risque de contenir des erreurs en comptant sur ses membres pour les corriger rapidement, et un système épistémique verrouillé qui vérifie les informations (par le choix d'experts) avant de les publier. C'est le débat de philosophie de la connaissance entre une conception de la rationalité permissive qui

4. La Wikipistemology de Fallis veut étudier les avantages et inconvénients épistémiques de Wikipédia du point de vue de philosophie de la connaissance. Les questions de la fiabilité et de la vérifiabilité des informations contenues par l'encyclopédie libre sont bien sûr abordées mais également les autres vertus épistémiques de cette encyclopédie telles que, pour n'en citer qu'une, la fécondité (cf. Thagard). 
compte sur l'autocorrection et la confiance épistémique et une conception de la rationalité rigide et restrictive qui demande d'abord de justifier ses croyances avant de les considérer comme connaissances.

Le constat du succès de l'encyclopédie permet de répondre à certaines critiques : Wikipédia est globalement fiable et a de nombreux avantages (cf. Fallis 2008). Pour autant, la pertinence de son système peut toujours faire l'objet de discussion. Par exemple, Sanger (2009) milite pour la création d'une place spécifique (privilégiée) pour les experts dans Wikipédia. En effet, Wikipédia n'est pas seulement " l'encyclopédie que tout le monde peut modifier » ( « the free encyclopedia that anyone can edit »). C'est aussi l'encyclopédie que tout le monde peut corriger : tout le monde est contributeur, tout le monde est rapporteur (Cardon et Levrel, 2009). Sanger considère que le succès de l'encyclopédie n'est pas le signe d'un bon modèle épistémique (mais plutôt de la qualité des contributeurs). Ainsi, le système égalitaire de Wikipédia ne serait pas épistémiquement vertueux et son succès pourrait être expliqué par des considérations plus sociologiques que philosophiques. Il est bien sûr possible de répondre à Sanger en mentionnant les échecs des encyclopédies en ligne faisant une distinction entre contributeur et expert (Citizendium, Veropedia, ...). Dans la plupart des cas, la communauté, dessaisie du pouvoir de décision, arrête de participer au projet. Mais un argument plus pertinent consiste à montrer l'existence d'une forme de reconnaissance de l'expertise dans Wikipédia (cf. Willaime 2015). De Laat, dans une étude comparative de procédures d'expertise d'encyclopédies, l'exprime différemment : au lieu de placer la confiance dans un expert a priori (avant que l'article ne paraisse), le statut d'expert digne de confiance de chaque contributeur (pour ceux qui sont effectivement identifiés) se construit petit à petit avec son historique de contribution, sa réputation fluctuante dans la communauté, au fur et à mesure de l'évolution de Wikipédia. C'est un statut dynamique, et c'est par ailleurs un statut directement lié au logiciel de wiki et à la logique d'archivage absolu et de transparence de Wikipédia (de Laat, 2012). Le débat porte ici non seulement sur la pertinence de certaines pratiques épistémiques mais surtout sur deux manières de concevoir la connaissance, deux modèles épistémiques entre importation d'une expertise préexistante et constitution d'une expertise spécifique à la communauté.

On peut donc comprendre les différentes communautés épistémiques en ligne comme autant de «modèles épistémiques ». Un modèle épistémique est alors un ensemble de comportements et de représentations conceptuelles explicites ou implicites au sein d'une communauté. Nous ne parlons pas ici d'un processus de modélisation qui consisterait à détacher les structures essentielles d'une réalité tout en la simplifiant suffisamment pour en permettre l'étude par des outils computationnels. Au contraire, le modèle épistémique repose sur une étude de cas. Celui de Wikipédia est fortement influencé par le logiciel MediaWiki qui structure l'encyclopédie. Ce modèle est souvent considéré comme procédural car il est basé sur le respect de certaines « procé- 
dures » considérées comme garantes de la fiabilité de l'encyclopédie (présence de sources par exemple). Par opposition, il n'est pas centré sur les agents : peu importe qui contribue, s'il respecte les règles, sa contribution est considérée comme valable et donc potentiellement épistémiquement pertinente. En philosophie de la connaissance, ce modèle procédural s'apparenterait au fiabilisme, cette position qui considère que c'est le processus de formation d'une croyance, fiable ou non, qui nous permet de la considérer comme une connaissance.

On pourrait donc considérer que le succès populaire de Wikipédia valide en quelque sorte la position philosophique qu'est le fiabilisme. Cependant, la réalité est plus complexe. Il est bien sûr possible d'expliquer le mode de fonctionnement de Wikipédia à l'aide d'une autre position épistémique. Certes le modèle épistémique explicite (c'est-à-dire les attitudes correspondantes au respect des règles) de Wikipédia correspondent au fiabilisme, mais on peut se demander si la réalité des usages (qui peuvent détourner les règles et ne pas respecter les recommandations de Wikipédia) correspond au fiabilisme. Willaime (2015) défend l'idée que le fiabilisme seul ne peut pas expliquer toutes les pratiques des wikipédiens et qu'il y a une évaluation (implicite) des contributeurs via, en particulier, des outils comme l'historique des modifications. L'analyse de modèle épistémique permet donc un aller/retour entre théorie épistémique et étude de cas. Elle ancre l'épistémologie sociale dans un contexte sans pour autant évacuer les questions d'épistémologie classique. Les questions théoriques sont contextualisées et peuvent avoir des conséquences en pratique.

Les possibilités techniques propres à l'informatique et l'émergence des cultures numériques ont donc contribué à motiver l'émergence d'une épistémologie 《sociale » attentive aux systèmes d'interaction et au caractère collectif de la connaissance. En étudiant le numérique, l'épistémologie sociale s'expose à la réfutation de certaines positions théoriques; une thèse concernant la nature de la connaissance peut en effet être mise à mal par l'étude de communautés épistémique telles que Wikipédia. Ainsi, la technique influence les positions épistémiques; notre deuxième partie montrer que l'inverse est également vrai.

\section{Wikipédia, mouvement du logiciel libre et ma- térialité}

Dans cette deuxième partie, nous allons tenter de montrer la nécessaire interdépendance du modèle épistémologique de Wikipédia et des aspects matériels et techniques. Ceux-ci s'inscrivent dans un contexte plus large et éminemment politique qui soulève un certain nombre de questions. 


\section{$2.1 \quad$ Études des sciences et techniques}

Décrire la révolution numérique, même si elle concerne les usages plutôt que les outils ne peut pas faire l'économie de l'influence du technique sur cette révolution. La matérialité est importante pour comprendre l'épistémologique. Pour donner corps à cette idée, nous nous tournons vers les STS (Science and Technology Studies, ou études des sciences et techniques) puisque ce sont les STS qui ont pour vocation de décrire l'influence réciproque du technique, de l'épistémologique, mais aussi du politique.

Ce n'est pas seulement que le technique est socialement construit (Bijker 2012), c'est aussi que le technique influence le social (« do artefacts have politics? », Winner 1980), et que des valeurs sont introduites dans la technologie.

Dans le cadre plus précis de l'informatique, l'ordinateur est une technologie qui n'y échappe pas. Dans un premier temps l'ordinateur a été objet de «Big Science » (c'est à dire d'une science des grands instruments), difficile d'accès pour ses utilisateurs, réservé à des spécialistes, symbole d'une science d'état, voire militaire, symbole aussi d'un pouvoir centralisateur, lié à une activité scientifique construite du haut vers le bas, perçu comme une incarnation de Big Brother, symbole aussi de l'interventionnisme de l'état... (Edwards 1997, Agar 2006).

Dans un deuxième temps il a été un objet banal, personnel, omniprésent, connecté, modulable, accessible, typique de l'époque du « scientific entrepreneur » (c'est à dire l'universitaire encouragé à créer une entreprise) (Aspray 2002), mais aussi vu comme instrument d'émancipation technologique individuelle (Turner 2008) avec l'aspect matériel de moins en moins limitant et les questions de logiciel (diffusion, licence,...) de plus en plus primordiales (Mahoney 2008, Campbell-Kelly 2006). Enfin, du point de vue des pratiques numériques, l'heure est aujourd'hui aux « big data " (l'exploitation quantitative de données massives) et à la « politique des algorithmes » (Cardon 2013, Gillespie 2014) : d'une part, la façon de produire les données, leur enregistrement, leur classement, sont liés aux formes que prend «l'infrastructure » informatique (Edwards 2007), d'autre part, les différents mondes du Web sont régis par les algorithmes à la base de leur définition de ce qu'est le monde numérique selon eux (de PageRank de Google à EdgeRank de Facebook), voire de ce que doit être le monde numérique quand ces algorithmes sont au cœur d'entreprises à vocation hégémonique (Gillespie 2014). Le PageRank de Google devient une "Weltanschuung », une vision du monde performative de ce que doit être le classement d'informations. Le EdgeRank de Facebook devient performatif des relations entre individus quand il sélectionne ce qui apparaît sur le mur de ces derniers. 


\section{$2.2 \quad$ Influences mutuelles}

De même, dans le cas de Wikipédia, l'influence réciproque de MediaWiki (le logiciel sur lequel est basé Wikipédia), du concept de wiki, et de l'encyclopédie Wikipédia est manifeste. Un wiki est une application web qui permet la création, la modification et l'illustration collaboratives de pages à l'intérieur d'un site web (Contributeurs Wikipédia, 2015). Le wiki a profondément bouleversé la façon d'appréhender le web. Imaginer (en 1995) un web où l'utilisateur peut lui même modifier la page qu'il consulte, plutôt que d'être obligatoirement passif vis à vis de ce que l'administrateur de la page lui propose est une révolution au sens où il pose les bases de ce qui sera appelé plus tard le web 2.0, un web vu comme un réseau où les internautes interagissent, plutôt qu'un web vu comme une bibliothèque que les internautes consultent.

L'histoire même du design de Wikipédia est notoirement liée au wiki puisqu'elle a démarré comme projet annexe d'une entreprise encyclopédique plus classique dans son rapport à l'expertise ${ }^{5}$ en attendant que cette dernière démarre... ce qui n'arrivera jamais.

C'est donc le wiki qui a permis l'idée de Wikipédia, et en retour le succès de Wikipédia a permis de populariser le concept de wiki à travers le web (Reagle 2010). Pour aller plus loin dans le technique, ce cercle vertueux expliquant le succès de Wikipédia en tant qu'entreprise encyclopédique faisant émerger sa propre épistémologie et celui du wiki en tant que représentation nouvelle du web, c'est aussi, du point de vue du logiciel, que MediaWiki est un logiciel libre, dont la licence est compatible avec sa dissémination, sa réutilisation, son amélioration par la communauté. De ce point de vue, les principes traduits en licence logicielle dans MediaWiki sont aussi traduits dans le domaine de la propriété intellectuelle dans l'établissement des licences Creative Commons qui régissent les droits d'auteur et les conditions d'utilisation de Wikipédia. (Lessig 1999) La référence aux Communs, déjà explicitée ailleurs (Cardon et Levrel 2009) se traduit dans le domaine juridique, dans le domaine procédural de l'administration de l'encyclopédie, et dans le domaine logiciel et tous ces domaines s'influencent mutuellement.

Évidemment, Wikipedia est une organisation qui possède sa propre bureaucratie. Certains contributeurs ont des pouvoirs spéciaux, comme celui de bloquer un article temporairement en cas de vandalisme répété par exemple. Ces contributeurs sont élus, leur réputation, voire leur éligibilité est liée à leur historique d'édition. Il s'agit donc d'une forme de méritocratie, mais la structure bureaucratique n'est pas définitive, elle n'est pas centralisée ni rigide (chaque Wikipédia dans chaque langue possède ses propres règles)

5. Le projet original des créateurs de Wikipédia, Nupedia, était une encyclopédie en ligne, mais écrite par des gens sélectionnés et écrivant des articles soumis à des rapporteurs. Le projet a été abandonné rapidement à la fois à cause de la faible croissance en nombre d'articles et du succès foudroyant du projet annexe Wikipédia. 
et, surtout, Cardon et Levrel insistent sur le fait qu'elle est basée sur la procédure plus que sur l'identité (Cardon et Levrel 2009). Konieczny décrit la gouvernance de Wikipédia comme adhocratique : décentralisation, faible contrôle hiérarchique, création de structures informelles et/ou temporaires. L'un des piliers de Wikipédia est même la limitation au possible des règles(Konieczny 2010). Ce qui ne veut pas dire que la structure ne possède pas ses rigidités : la communauté des Wikipédiens est liée par une idéologie commune, en particulier sur l'indépendance de Wikipédia vis à vis de toute influence extérieure, ce qui conduit à de violents débats quant à la question du problème de la faible représentativité des femmes dans la communauté, des méthodes à imaginer pour résoudre le problème, et de l'influence de cette faible représentativité sur le contenu et sur la structure de l'encyclopédie (Jemielnak, 2014). Au delà du wiki, Wikipédia est aussi administrée, dans ses règles, dans ses procédures, non seulement par la communauté mais aussi par des bots, c'est-à-dire des logiciels d'administration eux même gérés par la communauté, et qui participent à la définition de ce que doivent être les normes (de vérifiabilité, de neutralité, de violation de copyright, de gestion de la communauté) (Niederer 2010). Il y a donc un façonnage mutuel de la communauté, des règles et des logiciels. C'est la «politique des algorithmes » de Wikipédia.

Tkacz décrit par exemple comment les bots structurent le découpage des articles en chapitres, organisent les règles de politesse des pages de discussion, et ont ainsi une activité performative sur ce que doit être l'encyclopédie, et comment les échanges doivent se dérouler dans les pages de discussion (Tkacz, 2014). Niederer décrit comment l'activité des bots varie d'une Wikipédia à l'autre. Dans les Wikipédias de langue à faible nombre de locuteurs, la reconnaissance de l'encyclopédie passe par un nombre de liens optimal (pour pouvoir être référencée efficacement et ne pas disparaître dans les profondeurs de Google). L'activité des bots consiste dans ce cas à « linker» (à créer des hyperliens entre les différents articles et vers les articles dans d'autres langues) et représente parfois plus d'éditions que celles des contributeurs humains (Niederer 2010). Les bots définissent ainsi la structure de l'encyclopédie.

\subsection{Sphère publique}

Wikipédia c'est aussi une sphère publique au sens d'Habermas. La description habermassienne de Wikipédia par Dominique Cardon (Cardon et Levrel 2009) met en lumière le côté délibératif de l'encyclopédie en ligne. Selon Habermas, la sphère publique (c'est à dire, pour faire court, un endroit où l'on peut discuter sans contraintes de problèmes de société, et ce faisant, influencer la vie politique) doit garantir l'expression des membres quelque soit leur statut, ne pas être fermée, et partager des centres d'intérêt en commun. Ses membres ne doivent pas être aliénés par l'influence d'une autorité ou d'un pouvoir financier (Habermas 1991). La facilité pour accéder à l'information, 
pour la partager, la façon dont s'établissent les règles pour gérer le débat à l'intérieur de Wikipédia et pour se prémunir d'influences extérieures, font de la communauté des Wikipédiens un candidat moderne au statut de sphère publique à l'ère d'Internet (O'Sullivan 2009). Mieux, toutes ces dispositions sont intimement liées au dispositif sociotechnique qu'est le wiki, et les idéaux politiques liés à Wikipédia sont intimement liés aux questions de partage de la connaissance, y compris, et même surtout, du point de vue technique.

Il ne s'agit pas d'idéaliser la communauté des Wikipédiens, et, comme dans beaucoup situations pratiques émulant l'idéal d'une sphère publique, de nombreuses contraintes liées à la technique, mais aussi aux règles procédurales de la communauté nuisent à la réalisation de l'idéal (Auray 2009, Hocquet 2015). Dans le même ordre d'idées que les militants du logiciel libre ou ceux des biens communs, la communauté Wikipédia ressemble donc fort à ce que l'anthropologue Christopher Kelty a défini comme étant un «public récursif », ( « recursive public »), c'est à dire une sphère publique politiquement concernée par l'objet technique même par lequel elle s'exprime. Selon Kelty, l'objet technique du "public récursif » dépend de différentes couches successives imbriquées. Ces couches successives (logiciels d'application, compilateurs, langages, systèmes d'exploitation, matériel, protocoles Internet) sont toutes concernées d'une manière ou d'une autre par le débat politique au sein de la communauté (Kelty 2008).

\subsection{Public récursif}

Le travail anthropologique de Christopher Kelty consiste à s'intéresser à la question de savoir « comment » le mouvement/phénomène du logiciel libre marche, plutôt que savoir « pourquoi ». Kelty s'est intéressé aux pratiques des personnes impliquées dans le logiciel libre, plutôt que de tenter d'expliquer son (éventuel) succès. Il affirme qu'il y a cinq pratiques fondamentales qui définissent le public récursif du logiciel libre. Il s'agit bien de pratiques (et non de règles ou de normes), puisque d'une part, le travail de Kelty a consisté à observer en ethnologue les acteurs du logiciel libre dans leur milieu, et d'autre part, parce que Kelty estime plus pertinent, pour décrire les « gens du libre » de recourir à ce qu'ils ont en commun dans ce qu'ils font plutôt qu'à leurs discours.

Première pratique : partager le code source. La première pratique, inscrite comme principe de base par les acteurs du libre eux-mêmes, consiste à développer une communauté en partageant, donc en rendant ouvert le code source. Le fonctionnement du logiciel est ainsi accessible à tous.

Deuxième pratique : conceptualiser des systèmes ouverts. Pour construire un public récursif, le maximum d'objets technologiques doivent être ouverts, de l'ordinateur à l'Internet en passant par toutes les couches 
logicielles. Toutes ces technologies appartenant à des domaines différents sont rendues ouvertes chacune à leur façon (que ce soit les protocoles de transferts de données dans un réseau, comme le protocole TCP/IP, ou les systèmes d'exploitation des ordinateurs, comme GNU/Linux...). Il faut inventer une idée de ce qu'est « être ouvert » dans des domaines différents, pour que la communauté puisse évoluer dans un milieu adapté au public récursif.

Troisième pratique : écrire des licences. Pour chaque système ouvert conceptualisé, la façon de le partager et de l'inclure dans une des couches technologiques est d'en écrire la licence. Écrire des licences matérialise les conditions selon lesquelles les objets ouverts circuleront dans la communauté. Les licences d'utilisations de logiciels, particulièrement, sont la base du principe de leur diffusion.

Quatrième pratique : coordonner des collaborations. Comme le bazar décrit par Eric S. Raymond (1999), les communautés autour du logiciel libre ne sont pas caractérisées par leur organisation hiérarchique. Pour autant, mettre en œuvre des pratiques collaboratives fait partie de l'essentiel des mouvements du Libre. Les projets libres ne peuvent être viables que si la collaboration entre les acteurs est coordonnée. Cela n'implique pas forcément des organisations, mais pour le moins de l'adaptabilité.

Cinquième pratique : fomenter des mouvements. Les communautés du libre partagent aussi en commun la motivation de créer à partir de leur projet une façon de voir le libre. Construire une narration, voire être prosélyte, sont aussi des pratiques caractéristiques du libre.

Le parallèle avec les quatre libertés du logiciel libre de la Free Software Foundation est presque parfait, mais la caractérisation de Kelty est anthropologique : elle se base sur les pratiques. Il est intéressant de noter qu'à l'époque de « Two Bits » (Kelty 2008), Kelty englobe le mouvement Open Source et le mouvement Free Software dans la même communauté de pratiques, au même titre que Wikipédia ou d'autres mouvements liés indirectement au logiciel, mais que ses récents développements (Kelty 2013, Kelty 2014), considérant les aspects politiques, ne font plus l'amalgame entre les mouvements Free Software et Open Source (voir plus bas).

\subsection{Wikipédia en tant que public récursif}

La politique des acteurs du " libre » (c'est à dire toutes les communautés concernées par les pratiques décrites par Kelty, des contributeurs de Wikipédia aux développeurs de logiciels, en passant par la production de pair à pair ou « $\mathrm{P} 2 \mathrm{P} »)$ est donc concernée par la technique à la fois comme sujet de débat 
et comme moyen de débat. C'est le cas du wiki et de la communauté des Wikipédiens où tout est imbriqué : pas de Wikipédia sans wiki, pas de wiki sans neutralité du net, pas de neutralité du net sans système d'exploitation libre, ...

Wikipédia est un exemple paradigmatique d'un grand thème des STS : «Artefacts have politics ». On peut même rajouter «Artefacts have politics and epistemologies ». Les instruments ont une influence sur la connaissance, le wiki, les règles et les procédures de Wikipédia ont une influence sur l'épistémologie de Wikipédia. Ce n'est pas seulement le numérique qui permet à Wikipédia d'exister et de proposer de nouvelles choses à dire sur l'épistémologie, c'est aussi que le technique, Wikipédia et l'épistémologie qui en découle proviennent de choix politiques et que cette politique est directement liée au monde du « libre ».

Mais le monde du « libre » n'est pas politiquement univoque. Après avoir décrit les communautés dans son livre de 2008, Kelty en 2014 s'interroge sur la signification, voire la polysémie du mot « libre » dans ces communautés qui s'y réfèrent c'est l'objet « fog of freedom », de la liberté brumeuse (Kelty 2014).

\subsection{Liberté brumeuse}

Wikipédia est un représentant du « libre ». C'est le seul représentant du «libre» connu du grand public et c'est un des rares représentants du « libre » à grande portée en dehors du monde du logiciel. Par ces deux caractéristiques, Wikipédia est vue comme un porte drapeau d'un mouvement intiment lié au logiciel, mais qui veut s'étendre bien au delà du monde du logiciel, de l'ordinateur, d'Internet, de l'informatique et du numérique. Mais un représentant, un porte drapeau de quoi? A force de travailler sur les communautés du « libre », Kelty en est venu à interroger le sens politique de la « liberté » dans le monde du « libre ». Les STS permettent de relier le politique au technique, mais quelles théories politiques de la liberté se retrouvent dans le « libre » (Kelty 2014) ? De même Tkacz questionne le sens politique contenu dans l'« Open » (Tkacz 2012) des différents mouvements « Open » (Open data, Open science, Open government). Tous deux s'accordent à trouver ces mots polysémiques et poreux.

D'un côté, le logiciel libre tel que défini par la « Free Software Foundation » s'est au fil du temps fait de plus en plus le champion de la critique de la propriété intellectuelle, en particulier au nom de la liberté d'expression, de la vie privée, de la sécurité voire des valeurs individuelles du libéralisme (Kelty 2013). Le mouvement « Open Source », d'autre part, est lui plutôt concerné par des problèmes d'infrastructure, de management, d'entrepreneuriat, de concurrence, de maintenance, et de service après vente. Kelty en 2014 s'interroge sur ce que devient le « libre ». L'ordinateur personnel PC, l'Internet décentralisé (qui sont des infrastructures clés dans le développement du 
logiciel libre) disparaissent au profit d'infrastructures centralisées. L'informatique dans les nuages et ses serveurs gigantesques, la collecte centralisée de données individuelles, les matériels propriétaires de la téléphonie mobile (par opposition aux matériels standard typiques du PC), un Internet de moins en moins décentralisé et de plus en plus soumis aux barrières nationales, ... sont autant d'utilisations typiques de l'« Open Source » destinées à des actions centralisatrices, liées à des prétentions de monopoles.

Du système d'exploitation Android ${ }^{6}$ au Mechanical Turk d'Amazon ${ }^{7}$, les pratiques du public récursif ne sont plus respectées. L' " Open Source » est devenu un autre exemple de capitalisme absorbant sa critique (Boltanski 1999), en transformant une alternative politique (Le mouvement du « libre ») en un avatar néo-libéral (le mouvement « Open Source»).

Qu'en est il de Wikipédia dans ce cadre d'analyse? Les principes du bazar de Raymond (fondateur de la vision «Open Source» du « libre ») se sont détachés du monde du logiciel et sont devenus des mots-clés du Web 2.0 dans lequel on encourage la participation et la collaboration. Avec son principe « anyone can edit» (tout le monde peut contribuer), Wikipédia a remplacé Linux (et les logiciels en général) comme le nouveau parangon de l'« Open », dans le monde du Web 2.0. Nathaniel Tkacz utilise précisément Wikipédia comme étude de cas afin de comprendre ce qui se cache derrière "Open » (Tkacz 2012). D'un côté, une rhétorique politicienne absorbant le concept (comme par exemple, le soudain intérêt de beaucoup de politiciens pour l'Open Government) mais il y a plus : Tkacz y voit une filiation ramenant à Popper (et Hayek) qui définit la société « ouverte » par ce qu'elle n'est pas (un totalitarisme) plutôt que par ce qu'elle pourrait être (Popper 1945). Le problème de la société « ouverte » de Popper et de la vision libérale de Hayek et du Mont Pélerin telle qu'elle s'est ensuite réalisée est qu'elle produisait des problèmes (les monopoles, la propriété intellectuelle...) la rendant fermée, et c'est précisément dans le domaine du logiciel avec les problèmes de restrictions d'utilisation que les mouvements militants du « libre » sont apparus. Tkacz argumente donc que pour comprendre ce qu'est une «politique ouverte » ( «Open politics »), il est nécessaire d'observer la politique d'une communauté ouverte comme celle de Wikipédia dans sa pratique plutôt que dans son discours.

Par exemple, une pratique archétypale des communautés du logiciel libre est celle du «forking », c'est-à-dire de la possibilité (parfois menaçante) qu'une partie de la communauté se dissocie d'un projet ouvert lors d'un dissensus pour créer un projet concurrent en reprenant le code source du

6. Android est un système d'exploitation basé sur le système d'exploitation Linux mais qui dévoie les principes de GNU/Linux.

7. Le « Mechanical Turk» est une plateforme utilisant les principes du travail collaboratif chers au public récursif mais qui rejette l'égalité entre les contributeurs. Il se crée alors un rapport dissymétrique entre employeur et employé où le second perd tout pouvoir de décision et se contente de contribuer sans véritablement participer. 
logiciel initial. D'un coté la logique est violente, voire destructrice, et est une logique de compétition. De l'autre, cette menace est une réelle forme d' « empowerment », c'est à dire de capacitation de la base par rapport à la hiérarchie, de promotion de la méritocratie. L'histoire du «Spanish Fork », c'est à dire la scission de la Wikipédia hispanophone aux débuts de Wikipédia est un exemple de politique wikipédienne. A cette époque d'incertitudes quand aux financements pérennes de l'encyclopédie, les débats pour ou contre la publicité faisaient rage. Ce fork, la scission d'une partie de la communauté hispanophone, en faisant réaliser la menace d'implosion $\mathrm{du}$ projet a fait pencher la politique globale de financement de Wikipédia vers une absence totale de publicité. Étudier Wikipédia, dans l'évolution de ses règles, de ses procédures, de son organisation, de ses choix de licences, de ses bots, (comment distinguer ce qu'est une bonne participation d'une mauvaise participation, comment organiser la recherche du consensus...) c'est non seulement définir un certain «modèle épistémique », c'est aussi définir un modèle politique, héritier du logiciel libre dans un monde d'Open Source.

\section{Conclusion}

L'influence du numérique sur la formation de nos connaissances semble indéniable. Plutôt que de tomber dans un scepticisme souvent réactionnaire, il est nécessaire d'étudier précisément ces changements. Pour le philosophe de la connaissance, le numérique peut exemplifier l'importante grandissante des processus épistémiques collectifs. Ceux-ci demandent à être sérieusement pris en compte et analysés : c'est l'enjeu du développement récent d'une épistémologie dite « sociale ». Pour l'anthropologue du numérique, une conception politique transparaît de l'étude détaillée des règles, des procédures, des licences, des bots ou des formes de débats dans les communautés numériques. Les deux approches rassemblées montrent que l'étude des conceptions politiques du numérique est nécessaire pour qu'il puisse être épistémiquement utile.

\section{Références}

AgAR, Jon (2006), «What Difference Did Computers Make?», in : Social Studies of Science 36.6, ArticleType : research-article / Full publication date : Dec., 2006 / Copyright (C) 2006 Sage Publications, Ltd., p. 869-907.

Aspray, William (2002), "Computer Science and the Computer Revolution », in : The Modern Physical and Mathematical Sciences, sous la dir. de Mary Nye, Cambridge : Cambridge University Press, p. 598-614.

Auerbach, David (2014), «Encyclopedia Frown», in : Slate.

Auray, Nicolas et al. (2009), «La négociation des points de vue », in : Réseaux 154.2, p. 15. 
Berry, David M. (2012), « The Social Epistemologies of Software », in : Social Epistemology 26.3, p. 379-398.

BiJker, Wiebe E., Thomas P. Hughes et Trevor PInch, éds. (2012), The Social Construction of Technological Systems : New Directions in the Sociology and History of Technology, anniversary edition edition, Cambridge, Mass : The MIT Press, $472 \mathrm{p}$.

Boltanski, Luc et Eve Chiapello (1999), Le nouvel esprit du capitalisme, Edition : Essais, Paris : Gallimard, 843 p.

Campbell-Kelly, Martin (2004), From airline reservations to Sonic the Hedgehog : a history of the software industry, Cambridge, Mass. [etc.] : The MIT Press.

Cardon, Dominique (2013), «Présentation », in : Réseaux 177.1, p. 9-21.

Cardon, Dominique et Julien Levrel (2009), « La vigilance participative. Une interprétation de la gouvernance de Wikipédia », in : Réseaux 154.2, p. 51.

CoAdy, David (2011), «An Epistemic Defence of the Blogosphere », in : Journal of Applied Philosophy 28.3, p. 277-294.

- (2012), What to believe now : applying epistemology to contemporary issues, Chichester, West Sussex; Malden, MA : Wiley-Blackwell, 202 p.

Conein, Bernard (2007), « Technologies de la communication et épistémologie sociale », in : L'épistémologie sociale : une théorie sociale de la connaissance, sous la dir. d'Alban Bouvier et Bernard Conein, Collection Raisons pratiques. Épistémologie, sociologie, théorie sociale 17, Paris : Éditions de l'École des hautes études en sciences sociales, p. 191-210.

Contributeurs (2015), Wiki, in : Wikipédia, Page Version ID : 111182672.

Doueini, Milad. (2013), Qu'est ce que le numérique?, Paris : Presses universitaires de France.

Edwards, Paul N. (1997), The Closed World: Computers and the Politics of Discourse in Cold War America, The MIT Press, 462 p.

EDWARDS, Paul N. et al. (2007), « Understanding Infrastructure : Dynamics, Tensions, and Design », in : Final report of the workshop, "History and Theory of Infrastructure : Lessons for New Scientific Cyberinfrastructures".

Ensmenger, Nathan L. (2010), The Computer Boys Take Over : Computers, Programmers, and the Politics of Technical Expertise, The MIT Press, $336 \mathrm{p}$.

FALLis, Don (2008), « Toward an epistemology of Wikipedia », in : Journal of the American Society for Information Science and Technology 59.10, p. $1662-1674$.

Gillespie, Tarleton, Pablo J. Boczkowski et Kirsten A. Foot, éds. (2014), Media Technologies : Essays on Communication, Materiality, and Society, Cambridge, Massachusetts : The MIT Press, 344 p.

Goldman, Alvin (2008), " The social epistemology of blogging », in : Information technology and moral philosophy, p. 111-122. 
Goldman, Alvin I. (1979), «What is Justified Belief? », in : Justification and Knowledge, sous la dir. de George Sotiros PAPPAS, t. 17, Philosophical Studies Series in Philosophy, Springer Netherlands, p. 1-23.

- (1999), Knowledge in a social world, Oxford; New York : Clarendon Press; Oxford University Press.

HABERMAs, Jürgen (1991), The structural transformation of the public sphere : an inquiry into a category of bourgeois society, MIT Press, $332 \mathrm{p}$.

Hocquet, Alexandre (2015), "Wikipédia en tant que forum : une analyse de réseaux sociaux pour l'ethnographie de la production d'articles. », in : Wikipedia : objet scientifique non identifié, sous la dir. de Lionel BARBE, Louise Merzeau et Valérie Schafer, Intelligences numériques, Presses universitaires de Paris Ouest.

Jemielniak, Dariusz (2014), Common Knowledge? : An Ethnography of Wikipedia, Stanford, California : Stanford University Press, 312 p.

Kelty, Christopher (2013), « There is no free software », in : Journal of Peer Production 1.3.

- (2014), «The fog of freedom», in : Media Technologies : Essays on Communication, Materiality, and Society, sous la dir. de Tarleton GILlESPIE, Pablo J. Boczkowski et Kirsten A. Foot, Cambridge, Massachusetts : The MIT Press.

Kelty, Christopher M. (2008), Two Bits : The Cultural Significance of Free Software, Duke University Press, 402 p.

KoniecZny, Piotr (2010), «Adhocratic Governance in the Internet Age : A Case of Wikipedia », in : Journal of Information Technology 83 Politics 7.4, p. 263-283.

Krogh, Georg von et Eric von Hippel (2003), « Special issue on open source software development », in : Research Policy 32.7, p. 1149-1157.

LAAT, Paul B. de (2012), « Open Source Production of Encyclopedias : Editorial Policies at the Intersection of Organizational and Epistemological Trust », in : Social Epistemology 26.1, p. 71-103.

Lessig, Lawrence (1999), Code : And Other Laws Of Cyberspace, First Edition, New York : Basic Books, 320 p.

MaHONEY, M.S. (2008), « What Makes the History of Software Hard », in : IEEE Annals of the History of Computing 30.3, p. 8-18.

MÉLÈs, Baptiste (2013), « Unix selon l'ordre des raisons : la philosophie de la pratique informatique », in : Philosophia Scientice 17.3, p. 181-198.

Niederer, Sabine et José van Dijck (2010), « Wisdom of the crowd or technicity of content? Wikipedia as a sociotechnical system », in : New Media \& Society 12.8, p. 1368-1387.

O'Sullivan, Dan (2009), Wikipedia : a new community of practice?, Farnham, England; Burlington, VT : Ashgate Publishing, Ltd., 191 p.

Popper, Karl (1945), The Open Society and its Enemies : The Spell of Plato, New Ed edition, London : Routledge, $432 \mathrm{p}$. 
RAYMOND, Eric S. (1999), The cathedral \& the bazaar : musings on Linux and open source by an accidental revolutionary, 1st ed, Beijing; Cambridge, Mass : O'Reilly, $268 \mathrm{p}$.

REAGLe, Joseph Michael (2010), Good faith collaboration the culture of Wikipedia, Cambridge, Mass. : MIT Press.

SANGER, Lawrence M. (2009), " The fate of expertise after Wikipedia », in : Episteme 6.1, p. 52-73.

Stallman, Richard M.., Sam Williams et Christophe Masutti (2010), Richard Stallman et la révolution du logiciel libre, Paris : Eyrolles.

SUROWIECKI, James (2004), The wisdom of crowds : why the many are smarter than the few and how collective wisdom shapes business, economies, societies, and nations, 1st ed, New York : Doubleday, 296 p.

TkACZ, Nathaniel (2012), «From open source to open government : a critique of open politics », in : Ephemera : Theory and Politics in Organization Vol.12 (No.4), p. 386-405.

Turner, Fred (2008), From Counterculture to Cyberculture: Stewart Brand, the Whole Earth Network, and the Rise of Digital Utopianism, Chicago : University Of Chicago Press, $354 \mathrm{p}$.

WeBer, Steve (2004), The success of open source, Cambridge, MA : Harvard University Press.

Willaime, Pierre (2015), « Une analyse épistémologique de l'expertise dans Wikipédia », in : Wikipedia : objet scientifique non identifié, sous la dir. de Lionel Barbe, Louise Merzeau et Valérie Schafer, Intelligences numériques, Presses universitaires de Paris Ouest.

Winner, Langdon (1980), « Do artifacts have politics?», in : Daedalus, p. $121-136$. 